On the one hand there is new hope in the shape of

the thinking within the document; on the other

hand is the crying need for greater investment

and funding. . .

\title{
A delicate balance
}

The newly appointed Health Minister, David Lammy MP, has finally published Options for Change - the Chief Dental Officer for England's review of primary care dentistry in the NHS. In so doing, he has indicated the Government's determination to bring dentistry 'back into the mainstream of the NHS'. He has also said that he 'looks forward to working with the profession to make Options for Change a reality.'

The BDA has had a high level of input throughout the year-long gestation period of the Report and many of our ideas have been incorporated. The ideas in Options for Change came in the main from dentists, perhaps for the first time since the BDA Tattersall Committee's seminal report in 1964, which advocated wholesale reform of the NHS General Dental Service in particular. Since then there has been no shortage of reports, most of which are gathering dust on library shelves somewhere. We need to ensure that Options for Change does not suffer the same fate.

The report proposes options for change, which will explore alternative models for general dental practice that independent practitioners can take up on a voluntary basis. New ways of working and new ways of funding a service must evolve through an approach based on careful piloting and practical 'road testing' of new ideas, which will mainly come from dentists.

The need to increase the use of information and communication technology and move it beyond the simple EDI link to the Dental Practice Board to clinical applications as well as joining NHS Net is acknowledged in the report. It appears that funding for such a programme of improvement will be made available.

The concept of locally determined solutions for locally identified problems plays a major part in Options for Change. The BDA will continue to press the case for a dental presence on the Professional Executive Committee of all Primary Care Trusts and to promote the idea of dental networks pulling together groups of clinicians, NHS managers and patients to give NHS dental service delivery greater local ownership and cohesion.

The three Task Group reports attached to the main report provide a wealth of ideas on how to make improvements to NHS primary care dentistry. Many of these could be introduced immediately provided there are sufficient resources available, both human and financial. The report is for England only but it will also be read by the administrations in Scotland, Wales and Northern Ireland. Dentists in the Home Countries may in due course receive proposals for change along similar lines.

Volunteer dentists together with their Primary Care Trusts will now test some of the ideas in Options for Change through a process overseen by the NHS Modernisation Agency. If these 'field sites' work satisfactorily, the ideas can be rolled out to the profession and the NHS throughout England. Some changes could, following negotiation, be incorporated into the GDS contract and apply throughout the UK.

The Government admits that NHS primary care dentistry, both the General Dental Service and the Community Dental Service, is in poor condition. In the last month, BDA Chief Executive, Ian Wylie, described the situation as 'a dreadful indictment of neglect by successive governments'(BDJ 2002; 193: 124-125). Implementation will be driven by the profession, a process never before attempted, but one, which should reverse the neglect of recent years and bring benefits to dentists and the patients they serve.

The situation for NHS primary care dentistry is now finely balanced. There is new hope in the shape of the thinking within the document and new opportunities for dentists to seize. On the other hand is the crying need for greater investment and funding to persuade dentists that the NHS is, after all, a good system to work in. This process will test the Government's commitment to the modernisation of NHS dentistry by the amount of additional funding that finds its way into the service.

UK dentistry and the dental profession are standing at a major junction on their journey down the highway of modern healthcare. The dead end they are leaving behind is the now deeply distrusted and discredited 'fully comprehensive dental service for all, provided by the NHS'. One road ahead is that of exclusively private dentistry for that section of the population who are prepared to pay for it. The other road offers the profession an attractive mixed economy model that may be able to provide a new kind of service with something to suit everyone - dentists, patients and government. The situation is indeed delicately balanced and decisions taken now by all parties will have a lasting effect.

John Renshaw

Chairman BDA Executive Board 